nephron

Experimental

Nephrology

and Genetics
Nephron 2019;143:68-76

DOI: $10.1159 / 000501037$
Received: November 22, 2018

Accepted after revision: May 17, 2019

Published online: June 19, 2019

\title{
Mechanisms of Nephrogenesis Revealed by Zebrafish Chemical Screen: Prostaglandin Signaling Modulates Nephron Progenitor Fate
}

\author{
Brooke E. Chambers Rebecca A. Wingert \\ Department of Biological Sciences, Center for Stem Cells and Regenerative Medicine, Center for Zebrafish Research, \\ University of Notre Dame, Notre Dame, IN, USA
}

\section{Keywords}

Nephron · Segmentation · Zebrafish · Chemical genetics · Phenotypic screening $\cdot$ Prostaglandin

\begin{abstract}
Nephron development involves the creation of discrete segment populations that are specialized to fulfill unique physiological roles. As such, renal function is reliant on the proper execution of segment patterning programs. Despite the central importance of nephron segmentation, the genetic mechanisms that regulate this process are far from understood, in large part due to the experimental complexities and cost of interrogating these events in the mammalian metanephros. For this reason, forward genetics utilizing phenotypic screening in the zebrafish pronephros provides an avenue to gain novel insights about the mechanisms of nephron segmentation in the vertebrate kidney. Discoveries from zebrafish can highlight possible conserved pathways and provide a useful starting point for reverse genetic analyses with other animal models or in vitro approaches. In this
\end{abstract}

(c) 2019 S. Karger AG, Basel

E-Mail karger@karger.com www.karger.com/nef review, we discuss the results of a novel chemical screen using the zebrafish to identify segmentation regulators. Through this screen, we identified for the first time that prostaglandin signaling can modulate nephron segmentation, and that it is normally requisite during development to mitigate segment fate choice in the embryonic kidney. We briefly discuss how these discoveries relate to current knowledge about nephron segmentation. Finally, we explore the possible implications of these findings for understanding renal ontogeny and disease, and how this knowledge may be useful for ongoing research initiatives that are aimed at deciphering how to build or rebuild the human kidney.

(c) 2019 S. Karger AG, Basel

\section{Introduction}

Nephrons possess an intricate cellular composition, where unique differentiated populations occupy regionally distinct domains, known as segments. The precise order and arrangement of segments within the nephron 
is essential to kidney function [1]. Nephron segment composition is broadly conserved across vertebrate species, including fish, amphibians, reptiles, birds, and mammals [1]. In general, nephron anatomy consists of a blood filter, followed by a tubule that is comprised of a series of proximal, intermediate, and distal segments, where the last region is connected with the collecting duct system $[1,2]$. Differences in nephron segment composition reflect the variations between organismal physiology and habitat $[1,2]$. For example, avian and mammalian nephrons have a modified intermediate region, where the loop of Henle enables urine to be concentrated, and the length of this region varies dramatically among nephrons within each species, as well as between species $[1,2]$.

There have been continued advances in our knowledge about nephrogenesis in recent years due to complementary experimental studies in the zebrafish, frog, chick, and murine animal models, among others [2-4]. However, there is still much to learn about several aspects including segmentation, a term which refers to how nephrons are patterned along the proximo-distal axis, and how each specialized segment cell type is formed [2-4]. A deeper appreciation of the genetic regulation of nephron development is highly relevant to the origins and mechanisms underlying congenital abnormalities of the kidney [5]. Additionally, a greater command of nephrogenesis principles can be utilized to advance renal organoid research, where a current major challenge is the in vivo cultivation of functionally differentiated segment populations [6]. In this review, we discuss how our laboratory has leveraged the attributes of the zebrafish embryonic nephrons to perform a novel chemical screen that enabled the identification of regulators that affect nephron segment development and highlighted for the first time that prostaglandin signaling has essential roles during nephrogenesis in this vertebrate species.

\section{Mechanisms of Nephron Segmentation: Progress and Ongoing Challenges}

The process by which vertebrate nephron progenitors are fashioned into different segment populations has been the subject of growing research attention and has been reviewed in detail elsewhere [2]. Most recently, the molecular network for nephron segment patterning in the mammalian metanephros was shown to rely on a gradient of $\beta$-catenin activity that interacts with the BMP

Prostaglandin Signaling Regulates

Nephron Progenitor Fate pathway and Notch signaling [7], and levels of $\beta$-catenin also affect whether nephron progenitors differentiate or self-renew [8]. The interplay of these signaling pathways is thought to specify segment fates by controlling the expression of transcription factors, including Hnflb, Irx $1 / 2$, and Pou $3 \mathrm{f} 3$, for example $[9,10]$. Conservation of these mechanisms during nephron segment development has been suggested by comparative studies in Xenopus [9] and the zebrafish [11]. However, an understanding of the complete program of segmentation is far from complete. Knowledge gaps persist regarding the identity of the essential genes that pattern segment cell types or control their differentiation. Further, the mechanisms that govern boundary establishment between adjacent segment domains are not understood.

In part, these knowledge gaps are being addressed through efforts to fully characterize the transcriptome of every nephron cell. Recent technological advances in RNA sequencing have enabled detailed characterization of nephron cell-type expression signatures from adult and embryonic kidneys of both the mouse and human $[12,13]$. These exciting datasets proffer to reveal the inventory of genes that are requisite for developmental processes such as segmentation during nephrogenesis [12, 13]. Nevertheless, using these lists to further assemble the blueprint of instructions that build the nephron remains a long-range goal $[12,13]$. A key impediment to reaching this goal remains how to prioritize the selection of genes for loss and gain of function analyses. Once selected, researchers must complete the functional assessments of the individual genes, which involve considerable time and cost when conducted in mammalian models. In most cases, this is also likely to require strategies to conditionally abrogate gene function within the nephron and/or subsets of the nephron populace. The reason for such measures is that many renal factors are expressed at multiple stages of kidney formation and/or in other embryonic tissues, which precludes analysis of their contributions to nephrogenesis through standard knockout strategies. Further study of the genetic pathways will also involve design of approaches to facilitate the intricate work of delineating the relationships and connections between genes to assemble the regulatory networks that accomplish segment specification and differentiation. The continued implementation of cross-species analyses with other vertebrate models offers many valuable opportunities to conduct rapid and low-cost assessments of genes in these mammalian nephron transcriptome repositories. Such strategies can help to uncover functionally important molecular components, and thereby triage candidate 
genes of highest interest for further genetic studies in mammals.

Additionally, unbiased screening for nephrogenesis regulators through classical forward genetics and chemical genetics approaches affords the advantage of identifying important transcription factors and signaling pathways based solely on phenotypic outcomes. These forward approaches can identify novel components not evident from transcriptome data, and when convergent with such data can highlight the most likely candidates for the essential genetic components from among the dispensable. In particular, the zebrafish model is a powerful tool for unbiased phenotype screens to delineate the genetic pathways that control nephron development [14]. Zebrafish are vertebrates and possess high genetic similarity with humans [15]. Approximately $71 \%$ of human genes have a zebrafish orthologue [15]. Due to the teleost specific genome duplication, there are about 2 zebrafish genes for each human gene [15]. Therefore, study of human genes in the zebrafish must often consider multiple zebrafish orthologues, to determine whether redundant functions exist or subfunctionalization has occurred [15]. Despite these factors, several features of zebrafish embryogenesis facilitate their tractability for ontogeny research, including external fertilization, transparency during early development, and rapid organogenesis as most organs are formed over the first 24-72 hours post fertilization (hpf) [16]. These traits, along with fecundity and small adult size, support genetic studies because large numbers of animals can be generated and maintained with low costs and minimal husbandry space [16].

As such, the application of zebrafish for renal development [17-19] and disease studies [20-22] has grown tremendously in recent years. With respect to the kidney organ, zebrafish embryos develop an anatomically simple pronephros comprised of just 2 nephrons by $24 \mathrm{hpf}$ [23]. By this time point, the nephrons are fully segmented and contain a suite of cell types that are conserved with humans, including a series of proximal and distal tubule segments that display analogous expression profiles of the solute transporters that dictate the explicit physiological roles of each populace [24-26]. Zebrafish nephron segment composition is not identical to mammals, however, with notable differences in that they lack intermediate tubule segments that enable water conservation - an unnecessary task for a freshwater aquatic species [24, 25]. Nevertheless, the zebrafish model provides an opportunity to delineate the genetic mechanisms of glomerular ontogeny, as well as the processes that direct emergence of several tubule segments [24-27].

\section{Chemical Genetic Screen Links Prostaglandin Levels to Nephron Segment Patterning}

To expand our knowledge about the signaling pathways that affect nephron segmentation, we hypothesized that a chemical screen could help to identify nephrogenesis regulators $[28,29]$. We chose to implement a strategy utilizing a library of known bioactive molecules, the Screen-Well ICCB Known Bioactives Library (Enzo Life Sciences), which consists of nearly 500 compounds. The small molecules in the library include agents capable of affecting numerous cellular signaling pathways, and span such classes of compounds as kinase inhibitors, nuclear receptor ligands, protease inhibitors, and second messenger modulators [28]. The benefit of working with a known bioactive collection is that many or all aspects of the mechanism(s) of action for each compound have already been identified $[9,28]$. This enables the researcher to bypass many challenges in delineating the molecular target(s) of the compound and quickly determine how they can further study the "hits" isolated from the screen approach $[9,28]$. Therefore, our goal was to accomplish a comprehensive screen for agents that had the ability to alter nephron segmentation in vivo, which had the possibility of revealing essential molecular components of the normal segment development processes.

For the screen, we arrayed fertilized embryos into 96well plates and incubated them with either vehicle control or an experimental drug treatment from the library between the 4 and $24 \mathrm{hpf}$ time point [24] (Fig. 1a). The reasoning for this exposure window was that it overlapped with the entire period of time from when mesenchymal renal progenitors initially arise through the duration of their patterning into a pronephros organ comprised of 8 epithelial segment populations [24, 30, 31]. Embryo samples were then fixed for cell-type analysis, where whole mount in situ hybridization (WISH) was used to assess cell identities based on their expression signature of segment-specific transcripts. To gather information about multiple segments from each treatment, we used a multiplex WISH assay in which a cocktail of 3 cell-specific riboprobes was applied to detect 3 corresponding segment populations. The segments we screened were the podocytes, which contribute to the blood filter, the proximal convoluted tubule (PCT, the first of 2 proximal segments in the pronephros), and the distal early (DE, the first of 2 distal segments in the pronephros). Further, we selected these cell types for the multiplex WISH assay because they 


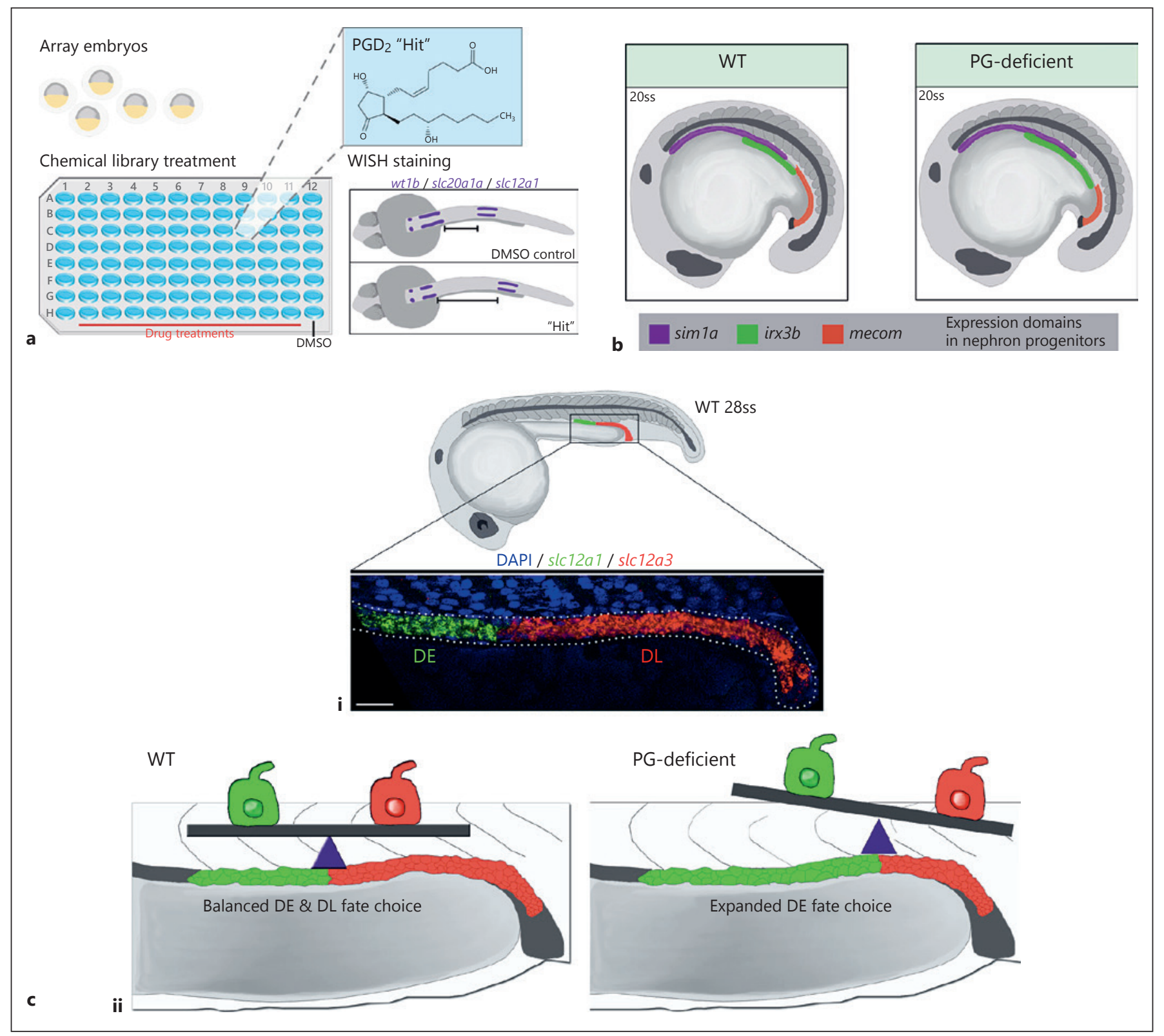

Fig. 1. Chemical screen in zebrafish embryos identified prostaglandin signaling as a modulator of distal segment fates in the developing pronephros tubule. a The screening strategy involved the physical array of small groups of WT zebrafish embryos into 96-well plates, after which individual drugs from the Screen Well ICCB Chemical Library were arrayed for experimental treatment during a time window relevant to pronephros genesis. b Schematic depicts how exposure to prostaglandin antagonists (e.g., inhibitors of ligand synthesis or receptor activity) led to changes in the expression domains of several essential transcription factors in the developing nephron progenitors compared to WT embryos, specifically sim 1a (purple), irx3b (green), and mecom (red) at the 20 somite stage (ss) of development. c i Nephrons within the zebrafish pro-

nephros organ have 2 distinct distal segments, the DE and DL marked by expression of the solute transporters encoded by $\operatorname{slc} 12 a 1$ and $s l c 12 a 3$, respectively. The confocal image shows a merged view of a fluorescent in situ hybridization experiment in which riboprobes were used to detect $s l c 12 a 1$ (green), slc12a3 (red), and DAPI staining (blue) to mark nuclei. Embryo illustration depicts the 28 somite stage of development, which is approximately $24 \mathrm{hpf}$. Scale bar $=20 \mu \mathrm{m}$. ii Prostaglandin signaling mitigates the balance between DE vs. DL segment fate choice in the zebrafish pronephros. In the absence of PG signaling, more nephron progenitors adopt a DE fate and fewer adopt the DL fate. WISH, whole mount in situ hybridization; WT, wild-type; DE, distal early; DL, distal late; ss, somite stage. 
comprise alternating nephron segments, which would facilitate clear evaluation of the 3 compartments and also allow for some indirect assessment of the intervening populations based on the physical appearance (size, distribution) of the populations that were being directly assessed.

From this approach, we scored approximately $16 \%$ of library compounds as "hits" associated with causing nephron phenotypes [24]. Each compound was recorded as to whether it had no effect at the dosage tested (e.g., wild-type) or was capable of promoting $(+)$ or inhibiting $(-)$ each discrete lineage $(\mathrm{P}+, \mathrm{PCT}+, \mathrm{DE}+)$ or $(\mathrm{P}-$, PCT,- DE-). The high percentage of flagged hits was expected given the multiplex nature of the assay that we employed, which was effectually 3 screens bundled together.

Of the hits obtained, there were numerous chemicals that corresponded to agonists or antagonists of the retinoic acid (RA) signaling pathway [29], which is an important regulator of development [32]. This result was especially encouraging to us, as it was a positive control from the vantage point that RA has been well established as an essential component of proximo-distal nephron segment patterning in the zebrafish [17, 24-26, 33-38]. RA is a vitamin A derivative that is processed into a secreted signaling molecule and binds to the RA receptors and retinoid $\mathrm{X}$ receptors, which are nuclear hormone receptors that effect transcriptional changes in response to interacting with an RA ligand [32]. Previous studies had demonstrated that elevating exogenous RA levels by incubating zebrafish with all-trans RA-induced proximal segments and inhibited distal fates, while inhibition of RA biosynthesis with antagonists or genetic mutations that disrupted RA production caused opposite effects [24]. RA-dependent segment changes were subsequently linked to alterations in the spatial domains of transcription factors that direct segment fates [33-35, 37, 38]. These include MDS1 and EVI1 complex locus (mecom), which is essential for the distal late (DL) fate [33], and SIM bHLH transcription factor 1 a (sim1a, formerly known as single minded 1a), which is essential for proximal straight segment (PST) fate [34]. From our segment screen, an example RA hit was the compound 4-hydroxyphenylretinamide, which is a synthetic analog of all-trans RA that expanded the proximal segments [29].

Upon surveying the other screen hits, we were fascinated to discover that agonists of the prostaglandin signaling pathway had scored as nephron segment modulators [29]. Prostaglandin signaling has important functions in a staggeringly broad range of normal biological contexts and disease states, in part due to the number and biochemical diversity of ligands and receptors [39, 40]. Prostaglandins are lipid mediators derived from arachidonic acid that can act in an autocrine or paracrine fashion through $G$ protein-coupled receptors to influence cellular activities like gene transcription [39]. The net effects of prostaglandin signaling include the control of physiological processes such as inflammation, pain perception, vasoregulation, and complex renal functions such as blood pressure control and fluid retention [39, 40]. In addition to these myriad roles, several recent studies have also uncovered important tasks for prostaglandin signaling in development. These advances were made possible using the zebrafish as they develop ex utero and had been masked in prior studies with mammalian models due to maternal contributions of prostaglandins to the fetus $[39,40]$. Developmental roles of prostaglandins are now known to include cell motility during gastrulation, angiogenesis, hematopoietic stem cell development, and binary fate choice between pancreas versus liver development [39-42]. Especially in light of its function in endoderm lineage decisions, we wondered if prostaglandin signaling similarly had a bona fide involvement in the cell fate choices of renal progenitors during nephrogenesis.

The prostaglandin moieties that had scored as hits in the screen corresponded to the ligands $\mathrm{PGD}_{2}, \mathrm{PGA}_{1}$, $\mathrm{PGJ}_{2}$, and $\mathrm{PGB}_{2}$ [29]. In each case, incubation with the prostaglandin during renal progenitor development led to a smaller PCT and a DE segment that was shifted caudally along the embryonic trunk, suggesting that a large intervening domain had formed-mostly likely corresponding to a larger PST. Because prostaglandins are notorious for possessing a short half-life, we sought to validate these screen hits by treating embryos with a long-acting derivative, 16,16 -dimethyl- $\mathrm{PGE}_{2}\left(\mathrm{dmPGE}_{2}\right)$ followed by WISH to assess the effects on each specific nephron segment. Interestingly, we found that the PST was indeed significantly expanded with exogenous $\mathrm{dmPGE}_{2}$, and that the DL segment, which is located caudal to the DE, was significantly reduced. These phenotypes occurred in the absence of other tissue defects, suggesting that renal development had been specifically affected. Further, we observed dosage-dependent effects of $\mathrm{dmPGE}_{2}$, as well as $\mathrm{PGB}_{2}$, on PST and DL segment size. These findings indicated to us that renal progenitors were exquisitely sensitive to alterations in prostaglandin levels in the embryo during nephrogenesis.
72

Nephron 2019;143:68-76 DOI: $10.1159 / 000501037$
Chambers/Wingert 


\section{Prostaglandin Signaling Mediates Fate Choice between Distal Segment Lineages}

Next, to explore whether prostaglandin signaling was actually a required component of normal nephron segmentation events, we performed loss of function studies [29]. Using parallel approaches of employing pharmacological inhibitors or gene knockdowns using morpholinos, we found that abrogation of the prostaglandin production machinery, in this case cyclooxygenase enzymes Cox-1 and Cox-2 (also known as prostaglandin-endoperoxide synthase 1 and 2a; Ptgs1, Ptgs2a, respectively), was sufficient to alter distal nephron segmentation. With disruption of Cox-1, Cox-2, or both enzymes, the DE segment was significantly expanded and the DL was significantly reduced. Further, we found that all of these disruptions could be rescued specifically by provision of $\mathrm{dmPGE}_{2}$. This indicated that $\mathrm{PGE}_{2}$ is likely the prostanoid that is required for pronephros development in the zebrafish.

Subsequently, we explored whether $\mathrm{PGE}_{2}$-mediated prostaglandin signaling was required in renal progenitors by assessing the spatial distribution of the known $\mathrm{PGE}_{2}$ G-protein-coupled receptors, prostaglandin E receptor 2a or 4a (Ptger2a, Ptger4a; also known as EP2 and $\mathrm{EP} 4$, respectively) [29]. We found that ptger $2 a$ and $p t$ ger4a transcripts were expressed in renal progenitors during somitogenesis stages, prior to the time when nephron segment pattern is established. These results were consistent with the hypothesis that Ptger2a and/or Ptger4a work in renal progenitors to affect their development.

To test this further, we abrogated each of these $\mathrm{PGE}_{2}$ receptors through parallel strategies involving genetic knockdown or pharmacological inhibition in zebrafish embryos and then assessed nephron segmentation by WISH. Similar to the Cox-1/Cox-2 studies, loss of Ptger2a or Ptger4a led to an expanded DE segment and reduced DL segment. These results further strengthened our interpretation that prostaglandin signaling was affecting the balance of the DE and DL segment fates during nephron development. To identify the developmental window when prostaglandin signaling affected segment fates, we performed loss or gain of function experiments with $\mathrm{dmPGE}_{2}$ and indomethacin, a nonselective Cox enzyme inhibitor, at different times of addition which enabled us to narrowly define the time of signaling [29]. Consequently, this led us to question how renal progenitors were altered during this developmental window that could lead to the DE/DL alterations.

Prostaglandin Signaling Regulates

Nephron Progenitor Fate
We explored the expression patterns of several essential transcription factors and discovered that their domains in the renal progenitor field were dramatically altered in response to changes in prostaglandin levels during this critical developmental window (Fig. 1b). For example, in prostaglandin-deficient embryos, the expression domain of Iroquois transcription factor irx $3 b$ was significantly expanded. As irx3b is required for DE fate [26], this result suggested that prostaglandin signaling regulates the irx $3 b$ domain to control DE fate choice. Subsequently, because Cox enzyme inhibition was unable to promote DE fate in irx $3 b$-deficient embryos, we were able to surmise that prostaglandin signaling works upstream of Irx $3 b$ to regulate the $\mathrm{DE}$ segment lineage.

Additionally, we found that the expression domain of mecom was reduced in prostaglandin-deficient embryos (Fig. 1b). Given that mecom is requisite for DL fate [33], this suggested to us that prostaglandin signaling promotes mecom expression within renal progenitors. However, we were unable to assess this relationship with additional studies because overexpression of mecom is toxic to embryogenesis [28] and would require engineering of a transgenic system to exact control over the spatiotemporal domain of mecom in renal progenitors.

Finally, in gain of function studies with $\mathrm{dmPGE}_{2}$ application, we discovered that the domain of the essential PST factor sim 1a [34] was significantly expanded. In contrast, sim 1a expression was unchanged in prostaglandindeficient embryos (Fig. 1b). This leads to effects on DE vs. DL fate (Fig. 1c). Taken together, this suggested the hypothesis that prostaglandin mediated promotion of $s i$ m1a underlies the expansion of the PST lineage when prostaglandin levels are elevated. In accordance with this, knockdown of simla was sufficient to abrogate PST expansion in $\mathrm{dmPGE}_{2}$-treated embryos.

\section{Mechanisms of Prostaglandin Signaling and Relation to Other Segmentation Pathways}

Our studies of renal progenitor development revealed that alterations in prostaglandin levels had potent effects on the regional gene expression domains of critical factors that mediate segment identities in the zebrafish pronephros. This suggests that prostaglandin signaling leads to patterning changes that specify the fate of the distal segment populations. However, more analyses are needed to ascertain whether these alterations involve cell proliferation, apoptosis, or changes in migration of the nephron progenitors. Furthermore, while our experi- 
ments have delineated that the transcription factors Irx $3 b$ and Mecom are key targets of normal prostaglandin signaling during zebrafish embryonic kidney development, and that elevating prostaglandin levels via exogenous $\mathrm{PGE}_{2}$ is sufficient to affect Sim1a expression in renal progenitors, we hypothesize that other genes are affected as well.

Continued efforts to determine the process of pronephros segment development using the zebrafish model can help to discover conserved pathways and mechanisms of segmentation. Toward this end, continued work is needed to uncover whether $\mathrm{PGE}_{2}$-mediated signaling has direct or indirect effects on the expression of $i r x 3 b$, mecom, and sim $1 a$ and other targets. For example, recent work has highlighted function of reciprocal antagonism between Sim 1a and Ppargcla that regulates PST segment size in the pronephros [43] and that Irx2 is a requisite for proper PST and DL segment development [44]. With regard to the DL segment, the T-box transcription factors $t b \times 2 a$ and $t b \times 2 b$ are essential to regulate DL fate [37]. Further, $t b \times 2 b$ acts downstream of mecom, and in turn the homeogene emx 1 acts downstream of $t b x 2 b$ to promote DL fate [45]. Interestingly, however, only emx1deficient embryos (not $t b \times 2 a / b$ or mecom) develop an expanded DE and reduced DL segment phenotype, which fully mimics the loss of prostaglandin signaling observed in the context of Cox enzyme or Ptger2a/4a deficiency [45]. Additionally, our recent research has revealed a cascade of Iroquois factors that interact with the transcription factor AP-2 alpha to coordinate a gene network that controls DE differentiation [46]. Interestingly, prostaglandin signaling also affects the fate choice between a transporter cell or multiciliated cell identify within the nephron [47]. Genetic studies that delineate the relationships between all of these nephron lineage networks will continue to grow our understanding of how nephron segmentation is controlled in the zebrafish, which provides inroads for parallel investigations in other vertebrates.

The larger question is whether prostaglandin signaling is a conserved mechanism that dictates nephron segmentation during renal development in mammals, and in particular whether it is relevant to human ontogeny or kidney disease. Whether prostaglandins have roles in mammalian development has not been ascertained in murine knockout studies to date because of maternal contributions - which has made the roles of this pathway historically underappreciated $[39,40]$. An intriguing place to test whether elimination of prostanoid production or receptor function has any effects on nephron segmentation would be in vitro systems such as organoid culture. Related to this topic, another aspect of interest is exploring how other mechanisms of nephron segmentation in the zebrafish, frog, and chick relate to mammals. For example, the Brn1 transcription factor has been linked for many years now to the establishment of distal segments in the developing murine metanephric kidney [48]. Conservation between mouse and zebrafish nephrogenesis has been suggested by the report that the zebrafish have 2 orthologues of Brn 1, pou $3 f 3 a$ and pou $3 f 3 b$, which are regionally expressed in the renal progenitors that give rise to the pronephros distal segments [26]. Future work to follow-up on such leads will cast further insight on the degree of conserved or divergent mechanisms by which vertebrate kidneys generate functional nephron segments.

\section{Summary}

There is an urgent need to identify innovative ways to repair, regenerate, and replace kidney functions for patients with congenital and acquired renal diseases. Knowledge about the mechanisms of nephron development can provide crucial insights for identifying new therapies for pathological conditions. Toward this end, information both about which molecules are sufficient to modulate nephrogenesis and how developmental programs normally control the process of nephron segmentation has many useful applications, such as enhancing the production of more biologically faithful renal organoids.

Our chemical screen for nephrogenesis regulators has led to the discovery that the prostaglandin signaling is normally required during nephrogenesis in the zebrafish embryonic kidney to dictate the balance between distal segment fates. Interestingly, alterations in COX-2 gene dosage in the mouse cause kidney development defects including hypothropic glomeruli and cortical thinning, though nephron segment ontogeny has yet to be assessed [49]. Only continued research on this topic will reveal whether prostaglandin signaling has conserved roles in nephron segmentation, and/or whether prostaglandin targets are conserved across any other vertebrates. Nevertheless, it is our hope that the research community can put to good use the information from our zebrafish screen, available within the publicly accessible database of zebrafish kidney chemical screen hits [29], to hunt for other key nephrogenesis mechanisms, or even to identify relevant pharmaceuticals for clinical applications.
Chambers/Wingert 


\section{Acknowledgments}

The authors thank members of the Wingert lab for helpful discussions and support.

\section{Statement of Ethics}

The authors have no ethical conflicts to disclose.

\section{Disclosure Statement}

The authors have no conflicts of interest to declare.

\section{Funding Sources}

This work was supported by the National Institutes of Health Research Grant R01DK100237 to RAW.

\section{Author Contributions}

B.E.C. and R.A.W. wrote and revised the article. B.E.C. created the original illustrations and performed the fluorescent in situ hybridization and confocal photography of distal segments in the zebrafish pronephros.

\section{References}

1 Romagnani P, Lasagni L, Remuzzi G. Renal progenitors: an evolutionary conserved strategy for kidney regeneration. Nat Rev Nephrol. 2013 Mar;9(3):137-46.

2 Desgrange A, Cereghini S. Nephron patterning: lessons from Xenopus, zebrafish and mouse studies. Cells. 2015 Sep;4(3):483-99.

3 McMahon AP. Development of the mammalian kidney. Curr Top Dev Biol. 2016;117:3164.

4 Little MH, Kumar SV, Forbes T. Recapitulating kidney development: Progress and challenges. Semin Cell Dev Biol. 2018 Sep; pii: S1084-9521(17)30426-3.

5 Schedl A. Renal abnormalities and their developmental origin. Nat Rev Genet. 2007 Oct; 8(10):791-802.

6 Oxburgh L, Carroll TJ, Cleaver O, Gossett DR, Hoshizaki DK, Hubbell JA, et al. (Re) Building a Kidney. J Am Soc Nephrol. 2017 May;28(5):1370-8.

7 Lindström NO, Lawrence ML, Burn SF, Johansson JA, Bakker ER, Ridgway RA, et al. Integrated $\beta$-catenin, BMP, PTEN, and Notch signalling patterns the nephron. eLife. 2015 Feb;3:e04000.

8 Ramalingam H, Fessler AR, Das A, Valerius MT, Basta J, Robbins L, et al. Disparate levels of beta-catenin activity determine nephron progenitor cell fate. Dev Biol. 2018 Aug; 440(1):13-21.

9 Heliot C, Desgrange A, Buisson I, PrunskaiteHyyryläinen R, Shan J, Vainio S, et al. HNF1B controls proximal-intermediate nephron segment identity in vertebrates by regulating Notch signalling components and Irx $1 / 2$. Development. $2013 \mathrm{Feb} ; 140(4): 873-85$.

10 Massa F, Garbay S, Bouvier R, Sugitani Y, Noda T, Gubler MC, et al. Hepatocyte nuclear factor $1 \beta$ controls nephron tubular development. Development. 2013 Feb;140(4):886-96.

11 Naylor RW, Przepiorski A, Ren Q, Yu J, Davidson AJ. HNF1 $\beta$ is essential for nephron segmentation during nephrogenesis. J Am Soc Nephrol. 2013 Jan;24(1):77-87.
12 Lindström NO, McMahon JA, Guo J, Tran T, Guo Q, Rutledge E, et al. Conserved and divergent features of human and mouse kidney organogenesis. J Am Soc Nephrol. 2018 Mar; 29(3):785-805

13 Lindström NO, De Sena Brandine G, Tran T, Ransick A, Suh G, Guo J, et al. Progressive recruitment of mesenchymal progenitors reveals a time-dependent process of cell fate acquisition in mouse and human nephrogenesis. Dev Cell. 2018 Jun;45(5):651-660.e4.

14 Lessman CA. The developing zebrafish (Danio rerio): a vertebrate model for highthroughput screening of chemical libraries. Birth Defects Res C Embryo Today. 2011 Sep; 93(3):268-80

15 Howe K, Clark MD, Torroja CF, Torrance J, Berthelot C, Muffato M, et al. The zebrafish reference genome sequence and its relationship to the human genome. Nature. 2013 Apr; 496(7446):498-503.

16 Lieschke GJ, Currie PD. Animal models of human disease: zebrafish swim into view. Nat Rev Genet. 2007 May;8(5):353-67.

17 Gerlach GF, Wingert RA. Kidney organogenesis in the zebrafish: insights into vertebrate nephrogenesis and regeneration. Wiley Interdiscip Rev Dev Biol. 2013 Sep-Oct;2(5):55985.

18 Drummond IA, Davidson AJ. Zebrafish kidney development. Methods Cell Biol. 2016; 134:391-429.

19 Naylor RW, Qubisi SS, Davidson AJ. Zebrafish pronephros development. Results Probl Cell Differ. 2017;60:27-53.

20 Poureetezadi SJ, Wingert RA. Little fish, big catch: zebrafish as a model for kidney disease. Kidney Int. 2016 Jun;89(6):1204-10.

21 Morales EE, Wingert RA. Zebrafish as a model of kidney disease. Results Probl Cell Differ. 2017;60:55-75.

22 Elmonem MA, Berlingerio SP, van den Heuvel LP, de Witte PA, Lowe M, Levtchenko EN. Genetic renal diseases: the emerging role of zebrafish models. Cells. 2018 Sep;7(9):E130.
23 Drummond IA, Majumdar A, Hentschel $\mathrm{H}$, Elger M, Solnica-Krezel L, Schier AF, et al. Early development of the zebrafish pronephros and analysis of mutations affecting pronephric function. Development. 1998 Dec; 125(23):4655-67.

24 Wingert RA, Selleck R, Yu J, Song HD, Chen $\mathrm{Z}$, Song $\mathrm{A}$, et al. The cdx genes and retinoic acid control the positioning and segmentation of the zebrafish pronephros. PLoS Genet. 2007 Oct;3(10):1922-38.

25 Wingert RA, Davidson AJ. The zebrafish pronephros: a model to study nephron segmentation. Kidney Int. 2008 May;73(10):1120-7.

26 Wingert RA, Davidson AJ. Zebrafish nephrogenesis involves dynamic spatiotemporal expression changes in renal progenitors and essential signals from retinoic acid and irx $3 \mathrm{~b}$. Dev Dyn. 2011 Aug;240(8):2011-27.

27 Kroeger PT Jr, Wingert RA. Using zebrafish to study podocyte genesis during kidney development and regeneration. Genesis. 2014 Sep;52(9):771-92.

28 Poureetezadi SJ, Donahue EK, Wingert RA. A manual small molecule screen approaching high-throughput using zebrafish embryos. J Vis Exp. 2014 Nov;93(93):e52063.

29 Poureetezadi SJ, Cheng CN, Chambers JM, Drummond BE, Wingert RA. Prostaglandin signaling regulates nephron segment patterning of renal progenitors during zebrafish kidney development. eLife. 2016 Dec;5:e17551.

30 Gerlach GF, Wingert RA. Zebrafish pronephros tubulogenesis and epithelial identity maintenance are reliant on the polarity proteins Prkc iota and zeta. Dev Biol. 2014 Dec; 396(2):183-200.

31 McKee R, Gerlach GF, Jou J, Cheng CN, Wingert RA. Temporal and spatial expression of tight junction genes during zebrafish pronephros development. Gene Expr Patterns. 2014 Nov; 16(2):104-13.

32 Duester G. Retinoic acid synthesis and signaling during early organogenesis. Cell. 2008 Sep;134(6):921-31. 
33 Li Y, Cheng CN, Verdun VA, Wingert RA. Zebrafish nephrogenesis is regulated by interactions between retinoic acid, mecom, and Notch signaling. Dev Biol. 2014 Feb;386(1): 111-22.

34 Cheng CN, Wingert RA. Nephron proximal tubule patterning and corpuscles of Stannius formation are regulated by the simla transcription factor and retinoic acid in zebrafish. Dev Biol. 2015 Mar;399(1):100-16.

35 Marra AN, Wingert RA. Epithelial cell fate in the nephron tubule is mediated by the ETS transcription factors etv5a and etv4 during zebrafish kidney development. Dev Biol. 2016 Mar;411(2):231-45.

36 Naylor RW, Skvarca LB, Thisse C, Thisse B, Hukriede NA, Davidson AJ. BMP and retinoic acid regulate anterior-posterior patterning of the non-axial mesoderm across the dorsal-ventral axis. Nat Commun. 2016 Jul;7(1):12197.

37 Drummond BE, Li Y, Marra AN, Cheng CN, Wingert RA. The tbx2a/b transcription factors direct pronephros segmentation and corpuscle of Stannius formation in zebrafish. Dev Biol. 2017 Jan;421(1):52-66.

38 Kroeger PT Jr, Drummond BE, Miceli R, McKernan M, Gerlach GF, Marra AN, et al. The zebrafish kidney mutant zeppelin reveals that brca2/fancd 1 is essential for pronephros development. Dev Biol. 2017 Aug;428(1): 148-63.

39 Cha YI, Solnica-Krezel L, DuBois RN. Fishing for prostanoids: deciphering the developmental functions of cyclooxygenase-derived prostaglandins. Dev Biol. 2006 Jan;289(2): 263-72.

40 Tootle TL. Genetic insights into the in vivo functions of prostaglandin signaling. Int $\mathrm{J}$ Biochem Cell Biol. 2013 Aug;45(8):162932.

41 North TE, Goessling W, Walkley CR, Lengerke C, Kopani KR, Lord AM, et al. Prostaglandin E2 regulates vertebrate haematopoietic stem cell homeostasis. Nature. 2007 Jun; 447(7147):1007-11.

42 Nissim S, Sherwood RI, Wucherpfennig J, Saunders D, Harris JM, Esain V, et al. Prostaglandin E2 regulates liver versus pancreas cell-fate decisions and endodermal outgrowth. Dev Cell. 2014 Feb;28(4):423-37.

43 Chambers JM, Poureetezadi SJ, Addiego A, Lahne M, Wingert RA. ppargcla controls nephron segmentation during zebrafish embryonic kidney ontogeny. eLife. 2018 Nov; 7:e40266.

44 Marra AN, Cheng CN, Adeeb B, Addiego A, Wesselman HM, Chambers BE, et al. Iroquois transcription factor irx2a is required for multiciliated and transporter cell fate decisions during zebrafish pronephros development. Sci Rep. 2019 Apr;9(1):6454.

45 Morales EE, Handa N, Drummond BE, Chambers JM, Marra AN, Addiego A, et al. Homeogene emxl is required for nephron distal segment development in zebrafish. Sci Rep. 2018 Dec;8(1):18038.

46 Chambers BE, Gerlach GF, Clark EG, Chen $\mathrm{KH}$, Levesque AE, Leshchiner I, et al. Tfap2a is a novel gatekeeper of nephron differentiation during kidney development. Development. 2019 Jun;pii:dev.172387.

47 Marra AN, Adeeb BD, Chambers BE, Drummond BE, Ulrich M, Addiego A, et al. Prostaglandin signaling regulates renal multiciliated cell specification and maturation. Proc Natl Acad Sci USA. 2019 Apr;116(17):8409_ 18.

48 Kopan R, Cheng HT, Surendran K. Molecular insights into segmentation along the proximal-distal axis of the nephron. J Am Soc Nephrol. 2007 Jul;18(7):2014-20.

49 Slattery P, Frölich S, Schreiber Y, Nüsing RM. COX-2 gene dosage-dependent defects in kidney development. Am J Physiol Renal Physiol. 2016 May;310(10):F1113-22. 